Abstract OP0215 - Table 1. Number (N), proportion of females (\%), and mean ages at baseline of incident patients with inflammatory arthritides between 2010 and 2014 in Finland.

\begin{tabular}{lccccc}
\hline & RA+ & RA- & UA & SpA & PsA \\
& $\mathbf{n}=\mathbf{6 1 8 6}$ & $\mathbf{n = 2 9 7 0}$ & $\mathbf{n = 2 9 5 9}$ & $\mathbf{n = 3 5 7 7}$ & $\mathbf{n = 2 6 7 0}$ \\
\hline Age, mean (SD) & $58(15)$ & $57(17)$ & $49(17)$ & $38(12)$ & $49(13)$ \\
$\begin{array}{l}\text { Proportion of females } \\
\text { (\%) }\end{array}$ & 66.4 & 67.2 & 67.4 & 52.3 & 48.8 \\
\hline
\end{tabular}

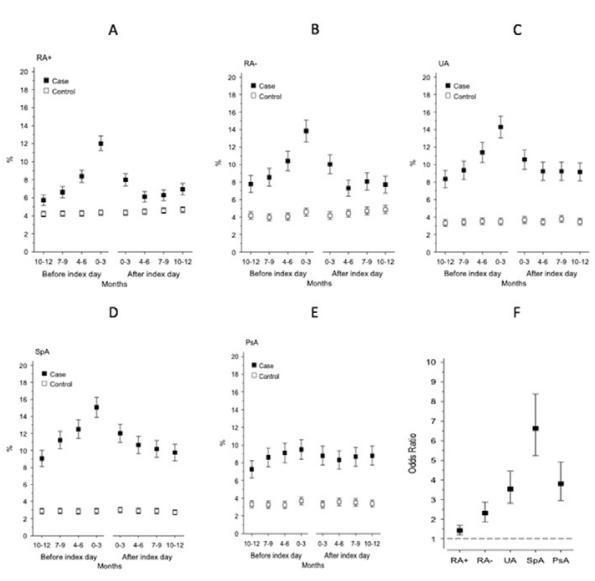

Abstract OP0215 - Figure 1. Proportion of opioid purchasers among patients with incident A) seropositive rheumatoid arthritis (RA+), B) seronegative rheumatoid arthritis (RA-), C) unspecified arthritis (UA), D) spondyloarthritis [(SpA), including ankylosing spondylitis and non-radiographic axial spondyloarthritis], and E) psoriatic arthritis (PSA) and their controls one year before and after the index date. F) Odds ratio for long-term opioid use among incident RA+, RA-, UA, SpA, and PsA patients versus controls after the index date.

Conclusions: IA patients are more likely to buy opioids one year before and one year after the diagnosis and presciption of antirheumatic medication than controls from the general population. The opioid purchases peak just before the index date in most IA patients. Long-term opioid use is also more common among patients with newly-onset IA, especially among those with SpA.

Disclosure of Interest: P. Muilu Grant/research support from: Competitive State Research Financing of the Expert Responsibility Area of Tampere University Hospital, Rheumatology Research Foundation, and Finnish Cultural Foundation., V. Rantalaiho Grant/research support from: Competitive State Research Financing of the Expert Responsibility Area of Tampere University Hospital, and Tampereen Reumayhdistys, H. Kautiainen: None declared, L. Virta: None declared, K. Puolakka: None declared

DOI: 10.1136/annrheumdis-2018-eular.4270

\section{OP0216 EXAMINING MODIFIABLE PSYCHOLOGICAL \& SOCIAL HEALTH FACTORS ASSOCIATED WITH USE OF OSTEOARTHRITIS ORAL ANALGESIC TREATMENT}

E.R. Vina ${ }^{1}$, L. Hausmann ${ }^{2}$, D.S. Obrosky ${ }^{2}$, A. Youk ${ }^{2}$, D. Weiner ${ }^{3}$, S. Ibrahim ${ }^{4}$, C. K. Kwoh ${ }^{1}$. ${ }^{1}$ Medicine, University of Arizona, Tucson; ${ }^{2}$ CHERP; ${ }^{3}$ Geriatric, VAPHS, Pittsburgh; ${ }^{4}$ CHERP, Philadelphia VAMC, Philadelphia, USA

Background: EULAR recommends the use of non-opioid oral medicines (acetaminophen, NSAIDs, or COX-2 inhibitors) for the management of knee osteoarthritis $(\mathrm{OA})$. Opioids are also recommended when these other therapies fail. There are known demographic and clinical differences in OA treatment use. Patients' social and psychological health may also influence use of medications, yet their association with the utilisation of opioid or non-opioid oral OA treatments is unknown.

Objectives: Determine which modifiable social and psychological health factors are associated with use of oral opioid and non-opioid medications for OA.

Methods: Baseline data from a randomised controlled trial that examined the effects of a positive psychological intervention on pain in veterans with knee OA were used. For our study, patients were categorised based on self-reported use of the following oral medications for OA at baseline: opioids (with/without other oral analgesic treatments), non-opioid analgesics, and no oral analgesic treatment. We used $\chi^{2}$ or analysis of variance to compare sociodemographic, clinical, social, and psychological health variables by baseline OA treatment use. We used multinomial logistic regression models to estimate adjusted relative risk ratios (RRRs) of using an opioid or a non-opioid analgesic (vs. no oral analgesic treatment), comparing patients by levels of social support (Medical Outcomes Study), health literacy ('How confident are you filling out medical forms by yourself'), and depressive symptoms (Patient Health Questionnaire-8). All models were adjusted for age, sex, race, income, OA symptom severity (WOMAC), self-reported Charlson comorbidity index, and body mass index.

Results: In this sample, $30.6 \%(n=110)$ reported taking opioid analgesics for OA $54.2 \%(n=195)$ reported non-opioid use, and $15.3 \%(n=55)$ reported no oral analgesic use. Compared to the other groups, those taking opioids were younger (mean age 62.5 vs 64.3 vs 67.1 , respectively, $p=0.002$ ) and had higher mean WOMAC scores (54.5 vs 45.7 vs $42.7, \mathrm{p}<0.001$ ). Opioid users also had lowe mean social support scores ( 10.0 vs 10.5 vs $11.9, \mathrm{p}=0.007$ ) and were more likely to have moderate-severe depression $(42.7 \%$ vs $24.1 \%$ vs $14.5 \%, p<0.001)$. Hav ing adequate health literacy did not differ by treatment group type.

Abstract OP0216 - Table 1 Social and psychological variables associated with oral analgesic treatment use*

\begin{tabular}{|c|c|c|c|}
\hline & $\begin{array}{l}\text { Adjusted relative } \\
\text { risk ratio }\end{array}$ & $95 \% \mathrm{Cl}$ & p-value \\
\hline \multicolumn{4}{|l|}{ Non-Opioid Oral Analgesic Use † } \\
\hline Social support & 0.94 & $(0.86,1.03)$ & 0.188 \\
\hline Adequate health literacy & 0.52 & $(0.21,1.30)$ & 0.160 \\
\hline Depression, moderate to severe & 1.93 & $(0.72,5.12)$ & 0.189 \\
\hline \multicolumn{4}{|l|}{ Opioid Use † } \\
\hline Social support & 0.92 & $(0.83,1.02)$ & 0.120 \\
\hline Adequate health literacy & 0.53 & $(0.20,1.42)$ & 0.207 \\
\hline Depression, moderate to severe & 2.96 & $(1.08,8.07)$ & 0.035 \\
\hline \multicolumn{4}{|l|}{ Opioid Use $\mp$} \\
\hline Social support & 0.98 & $(0.92,1.05)$ & 0.601 \\
\hline Adequate health literacy & 1.02 & $(0.55,1.89)$ & 0.954 \\
\hline Depression, moderate to severe & 1.53 & $(0.87,2.71)$ & 0.140 \\
\hline
\end{tabular}

The table 1 shows the associations between the social and psychological health measures with oral analgesic use, adjusted for sociodemographic and clinical factors. Having moderate-severe depression was associated with higher risk of opioid analgesic use compared to no oral analgesic use (RRR 2.96, 95\% Cl: 1.08 to 8.07) when adjusted for sociodemographic and clinical factors. Depression level was not significantly associated with non-opioid oral medication use, compared to no oral analgesic medication use, in a similarly adjusted model. Neither social support nor health literacy was associated with opioid or non-opioid ora analgesic use in fully adjusted models.

Conclusions: Knee OA patients with more severe depression symptoms, com pared to those without, were more likely to report using opioid (vs. non-opioid) analgesics for OA. Social support and health literacy were not significantly associated with oral analgesic use for OA when sociodemographic and clinical factors were accounted for

Disclosure of Interest: E. Vina: None declared, L. Hausmann: None declared, D. Obrosky: None declared, A. Youk: None declared, D. Weiner: None declared, S. Ibrahim: None declared, C. Kwoh Grant/research support from: Abbvie, EMD Serono, Consultant for: Astellas, EMD Serono, Thusane, Express Scripts, Novartis

DOI: 10.1136/annrheumdis-2018-eular.3813

\section{THURSDAY, 14 JUNE 2018}

\section{What is lupus - syndrome or different entities?}

\section{OP0217 A PERMEABLE BLOOD-BRAIN BARRIER IS NOT REQUIRED FOR NEUROPSYCHATRIC MANIFESTATIONS IN SLE AND PSS}

M.B. Lauvsnes ${ }^{1}$, A.B. Tjensvoll ${ }^{2}$, S.S. Maroni ${ }^{3}$, I. Kvivik ${ }^{1}$, T.B. Grimstad ${ }^{1}$, O. J. Greve ${ }^{4}$, E. Harboe ${ }^{1}$, L.G. Gøransson ${ }^{1}$, C. Putterman ${ }^{5}$, R. Omdal' ${ }^{1}{ }^{1}$ Medical Department, ${ }^{2}$ Neurology, ${ }^{3}$ Psychiatry, ${ }^{4}$ Radiology, Stavanger University Hospital, Stavanger, Norway; ${ }^{5}$ Division of Rheumatology, Albert Einstein College of Medicine, New York, USA

Background: A prevailing hypothesis for neuropsychiatric (NP) manifestations in systemic lupus erythematosus (SLE) and primary Sjögren's syndrome (pSS) is that brain reactive autoantibodies can enter the brain through an impaired bloodbrain barrier (BBB) during inflammatory conditions. Based on murine models the cytokine TWEAK could contribute to NP phenomena by binding Fn14 on brain endothelial cells. This will open the BBB and allow brain-reactive autoantibodies produced in the periphery to reach their targets in the brain.

Objectives: The aim of this study was to investigate the role of the BBB's permeability for NP manifestations in human SLE and pSS. Also, we wished to investigate whether increased TWEAK concentrations could be attributed to brain 
involvement as previously documented in the animal models of SLE. We compared TWEAK with markers of BBB permeability and astrocyte activation. Also, we estimated intrathecal B-cell activation, anti-NR2 abs, and explored whether these variables were associated with NP manifestations.

Methods: In a population-based cohort of 50 SLE (all fulfilling the ACR criteria) and $52 \mathrm{pSS}$ patients (all fulfilling the AECG criteria) NP manifestations were classified according to the ACR recommendations for NP-SLE. TWEAK, anti-NR2 antibodies (abs) were measured in serum and cerebrospinal fluid (CSF), S100b in CSF, and IgG index and Q-albumin were calculated.

Results: TWEAK concentrations in serum/CSF, as well as S100B and anti-NR2 abs in CSF, Q-albumin and IgG indices are shown in table 1. Associations between intrathecal TWEAK and S100B, Q-albumin and IgG index are given in table 2. No associations were found between TWEAK in serum/CSF and NP manifestations in the SLE, nor in the pSS group. Further, no associations were revealed between NP manifestations and S100B, Q-albumin or IgG index. AntiNR2 abs in CSF were associated with increased OR for dysfunction in the cognitive domains visuospatial processing (OR 4.9, $p=0.03$ ) and motor functioning (OR $6.0, p=0.006$ ) when corrected for age, gender, disease duration and education.

Abstract OP0217 - Table 1 Demographic and other selected data in 50 SLE- and 52 pSS patients

\begin{tabular}{llll}
\hline & SL.E & pSS & p-value \\
Age, years, mean (SD) & $43(12)$ & $57(12)$ & $<0.001$ \\
Females (\%) & $43(86)$ & $44(85)$ & 0.84 \\
Discase duration, years, mean (SD) & $12(9)$ & $7(5)$ & $<0.001$ \\
No with NP manifestations (\%) & $46(92)$ & $48(92)$ & 0.95 \\
TWENK, senum, pg/ml & $655(200-4644)$ & $925(451-1508)$ & 40.001 \\
TWEAK, CSF, pg/ml & $\$ 87(369-2351)$ & $1351(481.5184)$ & 4.001 \\
S100B in CSF, pg/ml & $221(110-420)$ & $264(138-544)$ & 0.005 \\
Q-albumin, ratio & $4.71(1.98-12.2)$ & $4.24(1.69-10.8)$ & 0.99 \\
IgG index, ratio & $0.17(0.10-0.39)$ & $0.18(0.09-1.12)$ & 0.19 \\
Anti-NR2 in CSF, ratio & $0.37(0.14-2.2)$ & $0.41(0.15 \cdot 3.0)$ & 0.20 \\
\hline Abbreviations Abs, & & &
\end{tabular}

Abbreviations: Abs, sntibodics

Anti-NR2 ab values given as a ratio against an internal calibrator with defined signal intensity.

Laboratory dats are presented as median and ranges

Abstract OP0217 - Table 2 Associations* between TWEAK in CSF and selected laboratory variables in 50 SLE- and 52 pSS patients

\begin{tabular}{lcclllll}
\hline & \multicolumn{3}{c}{ SLE } & & PSS \\
Dependent variables & $\beta$ & $\mathrm{R}^{2}$ & $p$-value & $\beta$ & $\mathrm{R}^{2}$ & $p$-value \\
\hline Q-albumin & 0.11 & 0.01 & 0.45 & 0.11 & 0.01 & 0.44 \\
$\mathrm{~S} 100 \mathrm{~B}, \mathrm{p} z \mathrm{~mL}$ & 0.50 & 0.25 & $<0.001$ & 0.45 & 0.20 & 0.001 \\
IgG index & -0.14 & 0.02 & 0.32 & 0.37 & 0.12 & 0.007 \\
\hline
\end{tabular}

Conclusions: Although several studies show that TWEAK seems necessary for CNS involvement in murine SLE, no clinical NP manifestations could be attributed to TWEAK concentrations in CSF/serum in the SLE- or pSS patients. Further, no associations were found between NP manifestations and the integrity of the BBB (Q-albumin), nor astrocyte activation.

The TWEAK concentration was higher in CSF than blood in both the SLE- and pSS patients, indicating an intrathecal production.

TWEAK in CSF covaried with S100B in CSF possibly reflecting a common ongoing intracerebral process.

We hypothesise that TWEAK is neuroprotective in human SLE and pSS. Brain residing immune cells produce brain reactive abs, for example anti-NR2 abs. These abs bind to neurons, and the cellular stress induced in the neurons leads to production of TWEAK. Concurrently, the activated B cells secret proinflammatory cytokines that among other actions activate astrocytes that in turn produce $\mathrm{S} 100 \mathrm{~B}$, also a neuroprotective protein.

Disclosure of Interest: None declared

DOI: 10.1136/annrheumdis-2018-eular.3923
THURSDAY, 14 JUNE 2018

\section{Genetics, epigenetics and disease: is it all in the genes?}

\section{OP0218 \\ INVESTIGATING THE REGULATORY SNPS AT THE RUNX3 LOCUS ASSOCIATED WITH ANKYLOSING SPONDYLITIS}

M.L. Vecellio ${ }^{1}$, A. Cortes ${ }^{2}$, C.J. Cohen ${ }^{1}$, R. Fischer ${ }^{3}$, B.P. Wordsworth ${ }^{1}{ }^{1}{ }^{\text {Nuffield }}$ Dept. of Orthopaedics, Rheumatology and Musculoskeletal Sciences; ${ }^{2}$ Wellcome Trust Centre for Human Genetics; ${ }^{3}$ Target Discovery Institute, University of Oxford, Oxford, UK

Background: Among the 100 genes associated with ankylosing spondylitis (AS), $R U N X 3$ a transcription factor (TF) involved in diverse immunological processes has a very robust $\left(10^{-15}\right)$ association. ${ }^{1}$ The biggest challenge following association studies remain to understand the mechanism behind this association and get insights of the disease. We have recently demonstrated that the association between AS and the single nucleotide polymorphism (SNP) rs4648889 located in a $2 \mathrm{~kb}$ regulatory locus upstream the promoter of RUNX3 can be explained by allele-specific effects on TF recruitment that alter gene expression, specifically in CD8 + T cells. ${ }^{2}$ In addition, another closely adjacent SNP, rs 4265380 shows functional effects (i.e. TF recruitment, histone marks enrichment and cell count) on CD14 +monocytes. ${ }^{3}$

Objectives: The main objectives of this work are: 1) to dissect the functional effects of the different SNPs at the RUNX3 locus, acting in different cell types (especially CD8 $+T$ cells and monocytes); 2 ) to identify the different interacting partners (i.e. TFs) binding at the RUNX3 locus in the presence of the AS-associated alleles.

Methods: We used publicly available dataset to define the epigenetic landscape of the RUNX3 locus. In vitro functional studies were performed to characterise the effects of these specific genetic variants, providing critical functional evidence for their role in AS.

Results: (1) Roadmap data revealed a robust peak for open chromatin and specific histone modifications associated with regulatory elements. Hi-C data showed the interaction of RUNX3 with different genomic loci within chromosome 1, in GM12878 lymphoblastoid cell line, CD8 +T cells and monocytes; (2) ChIP-qPCR experiments on monocytes and CD8 $+\mathrm{T}$ cells from AS patients revealed the enrichment for several histone modifications (i.e. H3K79Me2 and H3K4Me1) at the RUNX3 locus overlapping the SNPs of interest; (3) preliminary DNA pull-down experiments, followed by Mass Spectrometry, started to identify the whole range of proteins and TFs (DNA/protein 'interactome') that bind at the RUNX3 locus in the presence of the AS-associated alleles, evaluated in both CD8 +T cells and monocytes; (4) initial pathway enrichment analysis highlighted the distinct contribution of proteins involved in the transcriptional machinery (AS-risk VS protective alleles).

Conclusions: We provide first evidence that the $2 \mathrm{~kb}$ region upstream the RUNX3 gene has a plausible functional role in AS. These new observations are critically important not only in identifying specific cell types that play a pathogenic role in AS, but also in defining dysregulated pathways and potential therapeutic drug targets.

REFERENCES:

[1] ] IGAS, et al. Nat Genet 2013 Jul;45(7):730-8.

[2] Vecellio M, et al. Ann Rheum Dis 2016 Aug;75(8):1534-40.

[3] Vecellio M, et al. RMD Open 2018.

Disclosure of Interest: None declared

DOI: 10.1136/annrheumdis-2018-eular.2528 